\title{
Modelling physical injury to vehicle inhabitants - blast, fragment and acceleration environments resulting from the detonation of IEDs
}

\author{
F. A. Maestas \\ Applied Research Associates, Inc., USA
}

\begin{abstract}
Protective design for vehicle inhabitants against a terrorist attack on a vehicle and the resulting blast, fragment and acceleration environment requires a balanced understanding of the complex physical processes that occur and management of the inherent uncertainties associated with the modelling. This paper examines the key physics-based techniques required to accurately model the blast, fragment and acceleration environments on vehicles and their inhabitants that result from the detonation of an Improvised Explosive Device (IED). Specifically, this paper will outline techniques to model the environments that range from engineering equations to first principle approaches. Additionally, coupled and uncoupled approaches will be discussed. The resultant loads can then be used to model injury to the inhabitants of the vehicle. Loads on the inhabitants to be discussed are derived from primary blast, primary fragment, secondary fragment and acceleration based environments. Special emphasis will be placed on modelling techniques that result in acceleration loads on vehicle occupants.
\end{abstract}

Keywords: survivability analysis, modelling and simulation, personnel safety.

\section{Introduction}

The process used in most modelling tools for evaluating vehicle protective designs can be summarized with a simple acronym: PILR; Propagation, Interaction, Load and Response. This paper will discuss the PILR model as it applies to Improvised Explosive Devices (IEDs) on vehicles. Figure 1 provides 
this concept. Propagation is the environment that results from the detonation of an IED. Interaction describes how the environment interacts with the vehicle. Load refers to the load on the vehicle resulting from the detonation. Response is how the vehicle and contents, to include equipment and people, respond to that environment. Tools commonly used for the various areas are presented. A coupled approach will also be discussed briefly.

\section{Propagation}

An IED detonation environment can be divided into two primary aspects, blast and fragment. The acceleration environment will be addressed in the interaction section.

\subsection{Blast}

The level of fidelity in blast models varies somewhat from code to code. Key terms associated with describing a blast wave are illustrated in Figure 2. In an open space explosion, the blast wave is characterized by a discontinuous rise, called the "shock front" or "shock wave." Most weapon effectiveness or survivability models provide analytical approximations for the shock(s) that result from the detonations. These blast pressure time histories for both the static (side-on) pressure and dynamic pressure environments are evaluated. The peak pressures, time histories and the integration of the time history are used as loads on the vehicle, equipment and inhabitants. These blast models are generally only appropriate for conventional high explosives and are used to generate the ideal, free-field environment (Needham and Crepeau [1], Kingery and Bulmash [2]).

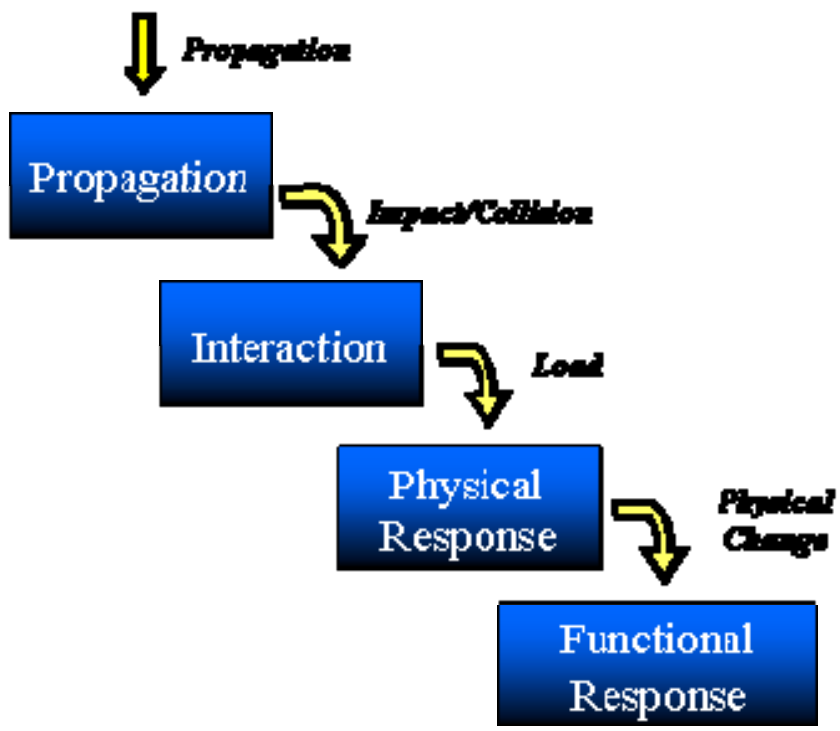

Figure 1: PILR representation of vehicle modelling. 
Overpressure - Static component of the blast wave. Increase in pressure over ambient conditions

Peak overpressure - Maximum overpressure value, at the shock front

Shock wave, shock front Discontinuous rise in overpressure at the arrival of the expanding gases

Blast wave - The pressure-time history, from arrival of the shock front to equilibration at ambient pressure

Positive phase impulse, impulse - Area under the positive phase of the overpressure-time curve

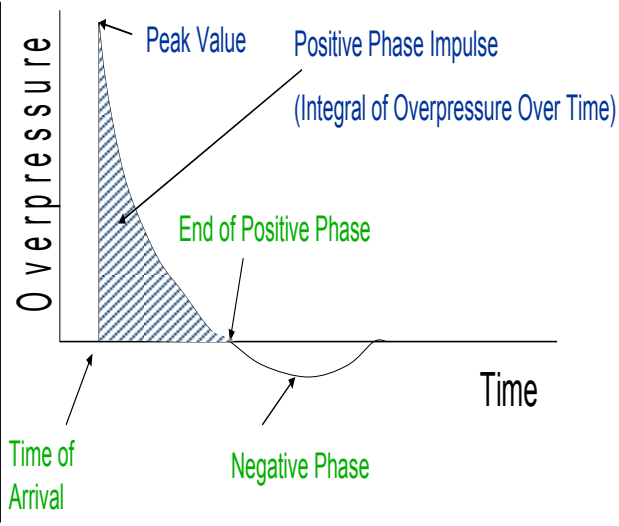

Figure 2: $\quad$ Pressure-time history of a blast wave.
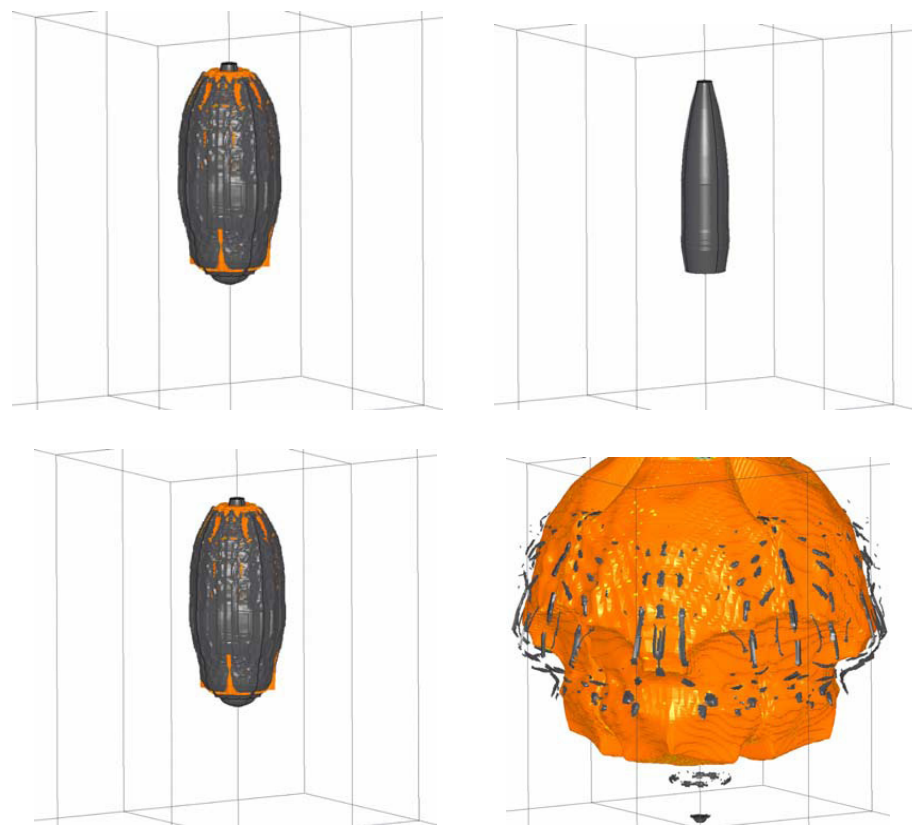

Figure 3: Initial IED detonation simulation.

\subsection{Fragments}

Many IEDs are constructed using unexploded inventoried ordnances. Thus, fragment fly-out can be modelled using a stochastically generated set of weapon fragments, based upon either Arena test data files or Mott's distribution [3]. Simplified algorithms can be used to determine fragment density, direction, mass and velocity. More detailed techniques such as finite elements methods are also used. As an example, Figure 3 provides a sequence of images from a finite 
element model of a generic IED threat (U.S.S.R. 152-mm Projectile Model OF540). In the model, a Lagrangian mesh was used for the case and an Arbitrary Lagrangian Eulerian (ALE) mesh was used for the high explosive (HE) fill inside the case and the surrounding air. The case material is shown in grey with explosive products shown in orange [4].

\section{Interaction}

Key to modelling the interaction of the environment with the vehicle is accurately modelling the vehicle. This requires more than a Computer Aided Design (CAD) representation. A common tool used is BRL-CAD [5]. Many times analysts translate a CAD representation into a finite element model. Figure 4 provides an example representation of an armoured personnel carrier. The personnel carrier is an M113A3 armoured transport vehicle. The LS-DYNA model was developed from a BRL-CAD representation.
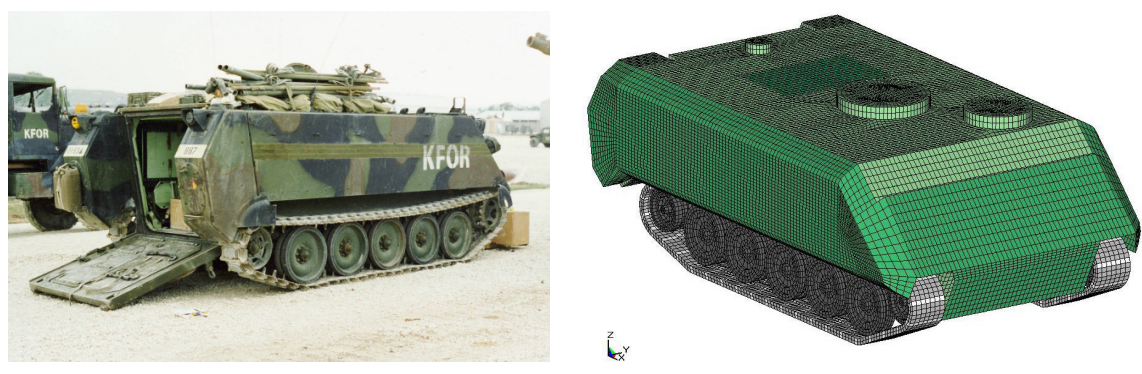

Figure 4: $\quad$ M113A3 armoured transport and LS-DYNA model.

\subsection{Blast}

The free field blast environment is significantly attenuated within the vehicle. Figure 5 illustrates the blast environment resulting from $38.75 \mathrm{lbs}$ of $\mathrm{C} 4$ detonated $10 \mathrm{ft}$ away from a vehicle similar in nature to that shown in Figure 4. Note the multiple reflections within the vehicle shown in the right graph as compared to the free field shown on the left [6].

\subsection{Fragment}

When an IED is constructed of a cased munition, fragment loading the vehicle have two effects: they impart momentum to the vehicle, contributing to the overall vehicle displacement, and they penetrate into the interior of the vehicle. Because the fragment penetration occurs at a slower rate than the blast loading, the breaches in the vehicle structure created by fragment penetration do not significantly facilitate blast wave propagation into the vehicle interior. Fragment penetration can be calculated using tools such as FATEPEN [7]. The 

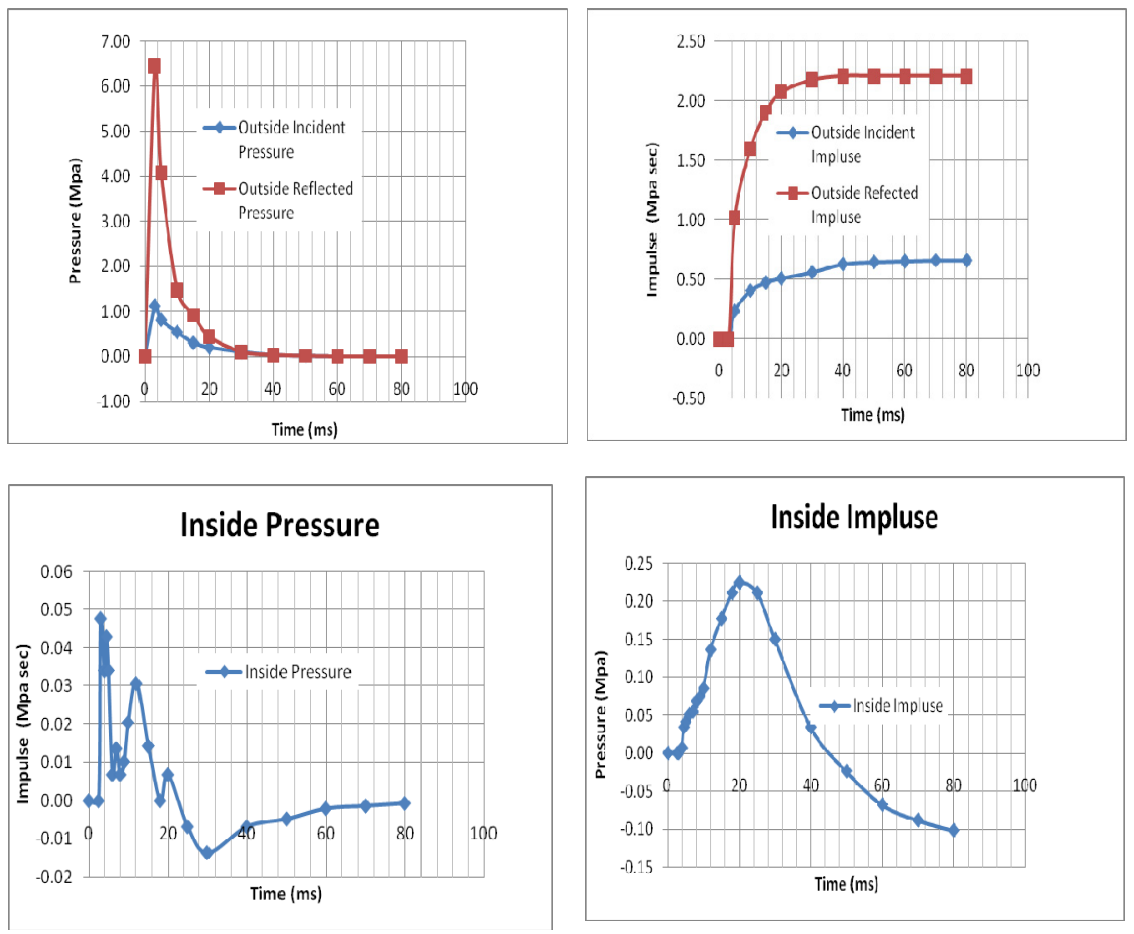

Figure 5: $\quad$ Blast overpressure and impulse inside and outside a vehicle.

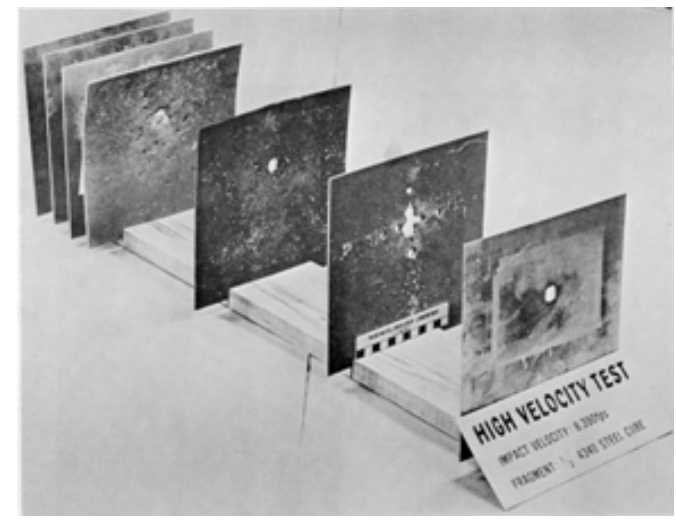

Figure 6: Multiple-plate penetration damage caused by a 240 grain steel cube impacting at $2.56 \mathrm{~km} / \mathrm{s}$.

significance of fragment penetration into the vehicle interior is the threat of penetration injuries to vehicle occupants. Figure 6 illustrates multiple plate penetration by fragments. 


\subsection{Acceleration}

Acceleration of the vehicle can be determined using engineering based tools such as BEAMS [8] or finite element based models such as LS-DYNA [9]. Vehicle acceleration is a function of the total impulse applied to the vehicle, which is heavily influenced, for buried detonations, by factors such as depth of burial, soil type and packing. Figure 7 illustrates the movement of a vehicle as the result of a land mine detonation.

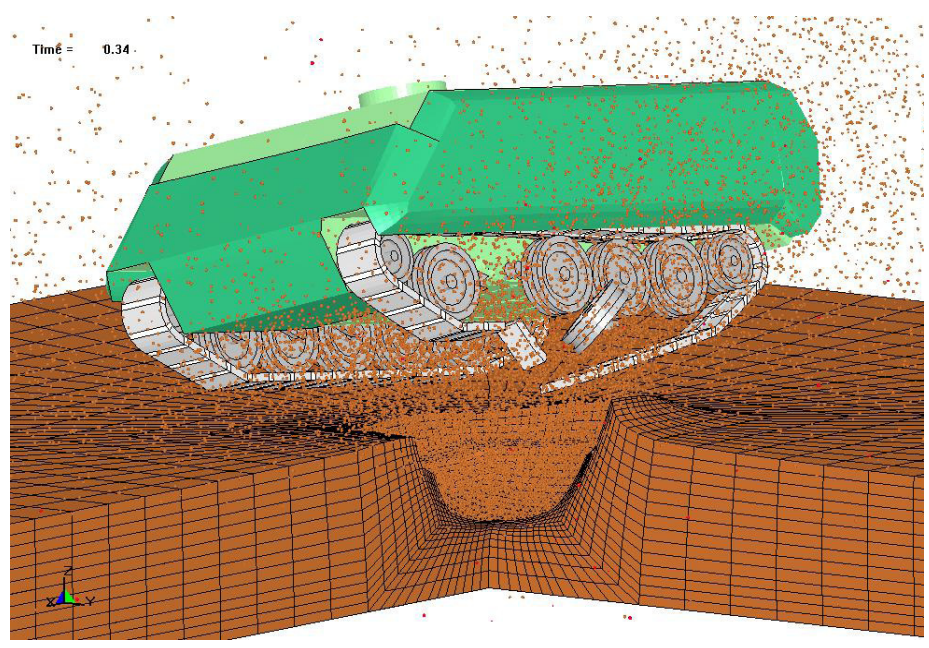

Figure 7: LS-DYNA SPH mine blast analysis at .34 second for a buried bare charge against an M113A3.

\section{Load}

The loads on the vehicle, equipment and inhabitants are calculated from the modified blast and fragment environments.

\subsection{Blast}

The blast load is calculated as a function of the distance (range) from the detonation point to the component of interest. The impulse is calculated by integrating the pressure time history over the area of interest. Peak pressure and impulse are the primary criteria for evaluating blast loading experienced by the crew.

\subsection{Fragment}

The fragment load is also calculated by integrating the impulse over the structural element. For equipment and personnel the load is typically momentum based. 


\subsection{Acceleration}

The blast event results in two dominant loadings to occupants; short-term localized floor and seat deformations and longer-term rolling and pitching of the vehicle at high rates. The relative magnitude, timing, and duration of the local and global motions will affect occupant survivability. As an example, the response of an M113 to a mine blast beneath a tread is shown in Figure 8. The occupant response is also shown in the figure for unbelted occupants where the vehicle structure has been made semi-transparent. Injuries associated with whole body acceleration are typically blunt impact type injuries, and are heavily influenced by the use or lack of use of occupant restraint systems (seatbelts) and protective gear.

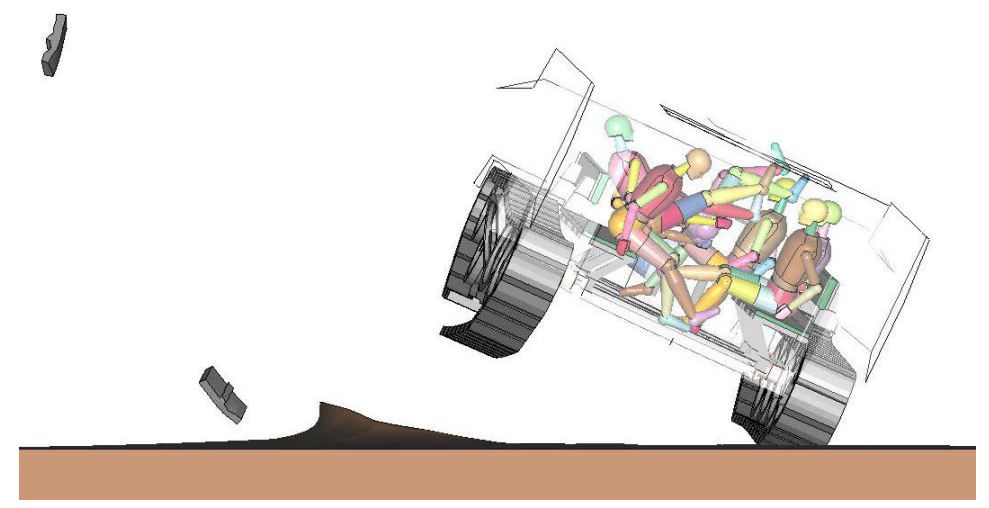

Figure 8: $\quad$ M113 mine blast simulation with occupants [4].

\section{Response}

When a detonation occurs near a vehicle, the resulting blast propagates through the air toward that vehicle. When it reaches the vehicle, momentum is imparted to that vehicle, and the blast both reflects off the vehicle and diffracts around the vehicle. The momentum imparted to the vehicle causes the vehicle, or components of the vehicle to accelerate, which results in either vehicle components or the entire vehicle to be displaced. Vehicle occupants may be subjected to blast and fragment environments if the vehicle shell is breached. Regardless of vehicle breach or not the inhabitants are subjected to acceleration. Resultant injury and/or death is a function of the loads that are experienced. An injury criterion is a "transformation function" which equates a measurable environmental condition or surrogate response to injury [10].

\subsection{Blast}

In some cases, injury criteria will yield a binomial response: injury versus no injury, or no injury versus death. But, in most cases, injury criteria define a 


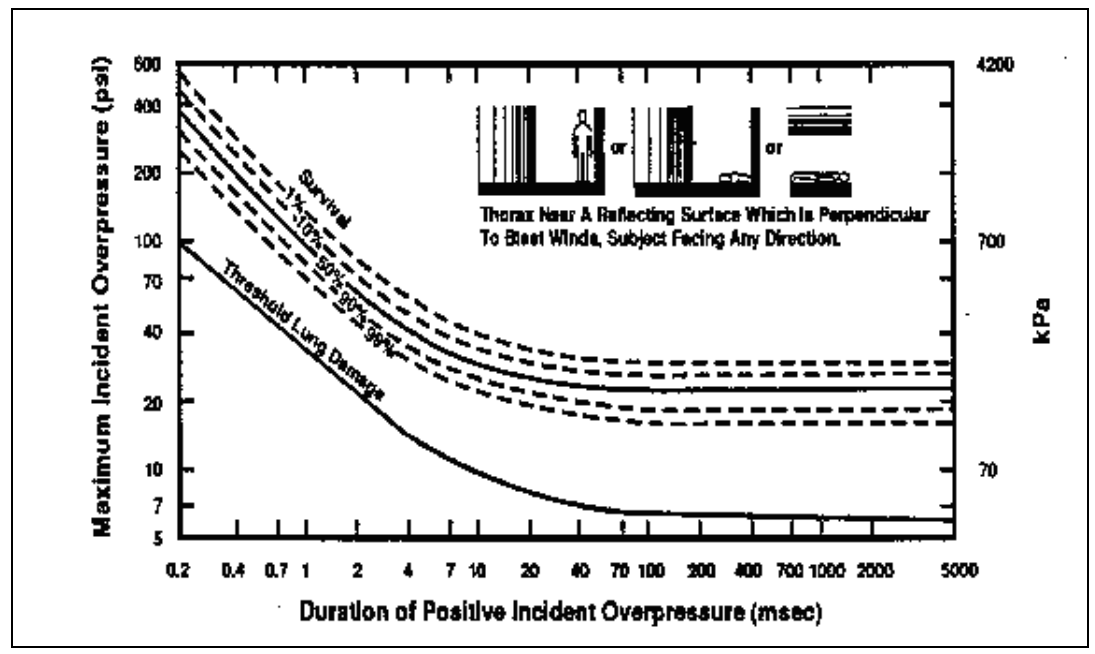

Figure 9: $\quad$ Bowen survival curves predicted for 70-kg man [11].

parameter space spanning from no injury through fatality. Figure 9 provides an example of the response for blast environments.

\subsection{Fragment}

For fragment environments, the same type of "transformation function" is required. Typically analysts use the Sperazza-Kokinakis Skin Penetration Threshold Model [12]. The Kokinakis Skin Penetration equation (eqn. (1)) uses debris velocity and debris area-to-mass ratio in determining which debris fragments are likely to cause skin penetration injuries.

$$
V_{\min }=125\left(\frac{\text { Area }}{\text { Mass }}\right)+22
$$

where:

$\mathrm{V}_{\min }=$ Minimum debris fragment velocity for skin penetration in $\mathrm{m} / \mathrm{s}$, Area $=$ debris fragment area in $\mathrm{cm} 2$,

Mass $=$ debris fragment mass in grams

The Area and Mass for a given debris fragment from a cloud are inserted into eqn. (1) to determine the $V_{\text {min }}$ required for skin penetration. Debris fragment impact velocities greater than $\mathrm{V}_{\min }$ (above the graphed line in Figure 10) are most likely to result in skin penetration injuries; debris fragments with velocities less than $\mathrm{V}_{\text {min }}$ (below the graphed line) are most likely to result in blunt trauma type injuries.

\subsection{Acceleration}

The acceleration based injury experienced by vehicle occupants can be attributed to injury to the head or the vulnerable organs of the thorax or upper abdomen. 
Data for evaluating injury comes from either the car industry or from tests characterizing the severity of injuries from falls. One measure that we can use to compare to the observational data is the $\mathrm{V}_{50}$, the velocity at which we expect $50 \%$ lethality. For a $75 \mathrm{~kg}$ man (thorax mass $17.25 \mathrm{~kg}$, contact area 400 square $\mathrm{cm}$ ) the $\mathrm{V}_{50}$ is calculated from the model to be about $14.6 \mathrm{~m} / \mathrm{s}$. Table 1 lists some $\mathrm{V}_{50}$ estimates for skull fracture and lethality in falls onto a hard flat surface. Entries are listed for the usual $75 \mathrm{~kg}$ man as well as a $55 \mathrm{~kg}$ woman.

\section{Coupled approach}

Modelling the effects of a mine blast on a vehicle involves modelling the blast source, coupling the loading from this source to the vehicle structure, and simulating the vehicle response. A 'fully' coupled analysis provides simultaneous solution of fluids, soil, and structural response for as much as every time step of a computational analysis. A simulation based design (SBD) approach [4] under development for the U.S. Army applies numerical methods which allow for the rapid iterative evaluation of various parameters in a coupled manner.

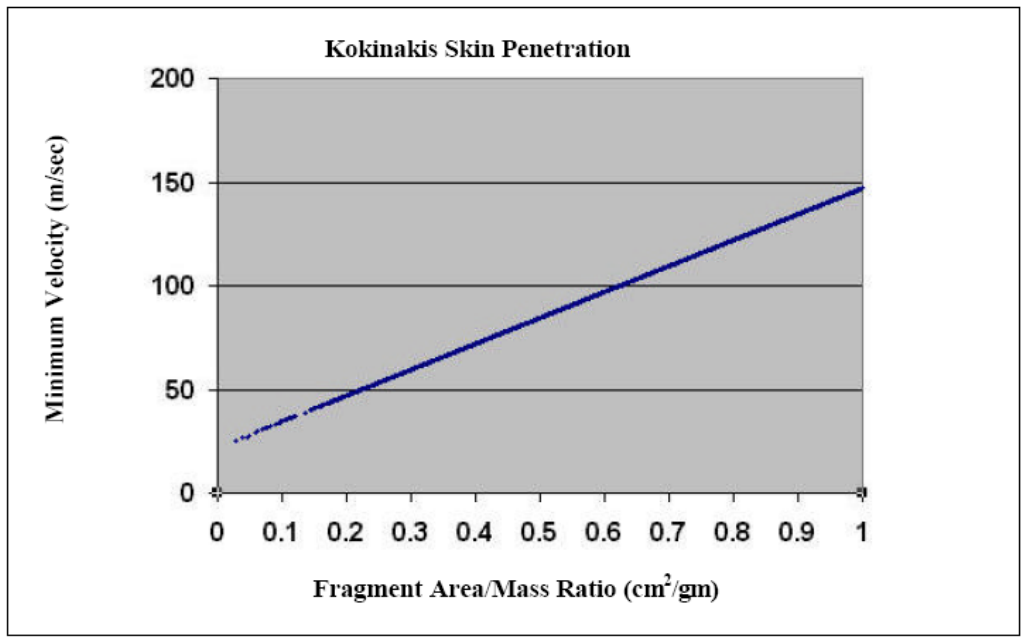

Figure 10: Fragment survival curves.

Table 1: $\quad$ Mean velocities for skull fracture and lethality in falls [13].

\begin{tabular}{|c|c|c|c|c|c|}
\hline Individual & $\begin{array}{c}\text { Mass of } \\
\text { Head }\end{array}$ & $\begin{array}{c}\text { Effective } \\
\text { Diameter, D }\end{array}$ & $\begin{array}{c}\text { Skull } \\
\text { Thickness }\end{array}$ & $\begin{array}{c}\text { V50 } \\
\text { (fracture) }\end{array}$ & $\begin{array}{c}\text { V50 } \\
\text { (lethal) }\end{array}$ \\
\hline $75 \mathrm{~kg}$ male & $4.5 \mathrm{~kg}$ & $5.7 \mathrm{~cm}$ & $5 \mathrm{~mm}$ & $6.9 \mathrm{~m} / \mathrm{s}$ & $12.4 \mathrm{~m} / \mathrm{s}$ \\
\hline " & " & " & $7 \mathrm{~mm}$ & $8.1 \mathrm{~m} / \mathrm{s}$ & $14.7 \mathrm{~m} / \mathrm{s}$ \\
\hline $\begin{array}{c}55 \mathrm{~kg} \\
\text { female }\end{array}$ & $4.0 \mathrm{~kg}$ & $5.6 \mathrm{~cm}$ & $4 \mathrm{~mm}$ & $6.5 \mathrm{~m} / \mathrm{s}$ & $11.7 \mathrm{~m} / \mathrm{s}$ \\
\hline " & " & " & $6 \mathrm{~mm}$ & $7.9 \mathrm{~m} / \mathrm{s}$ & $14.3 \mathrm{~m} / \mathrm{s}$ \\
\hline
\end{tabular}




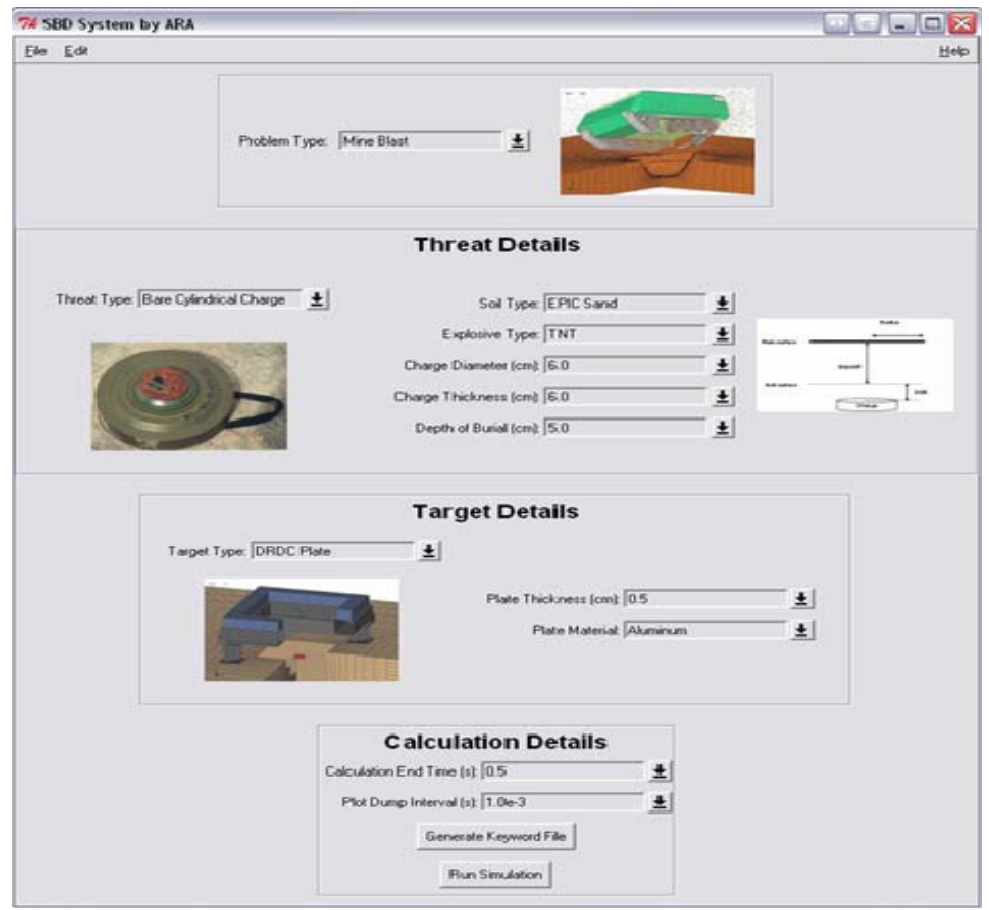

Figure 11: $\quad$ SBD graphical user interface.

\section{Conclusion}

Modelling of an IED detonation adjacent to a vehicle requires a balanced understanding of the complex physical processes that occur and management of the inherent uncertainties associated with the modelling. This paper provided the key physics-based techniques required to accurately model an IED attack on a vehicle. Clearly there was insufficient space to cover all the nuances of the modelling. However, the blast and fragment environment that results from an IED detonation, the loads on the vehicle and its occupants and the response of those occupants were covered in a manner to allow a basic understanding of the approach.

\section{References}

[1] Needham, C.E. \& Crepeau, J.E., The DNA Nuclear Blast Standard (1KT), DNA 5648T, prepared by S-Cubed for the Defence Nuclear Agency, Alexandria, VA, 1981.

[2] Kingery, C.N. \& Bulmash, G., Airblast Parameters from TNT Spherical Air Burst and Hemispherical Surface Burst, Technical Report ARBRL-TR- 
02555, U.S. Army Armament Research and Development Centre, Ballistic Research Laboratory, Aberdeen Proving Ground, MD, 1984.

[3] Grady, D., "The Statistical Fragmentation Theory of N. F. Mott", Shock Compression of Condensed Matter- 2003: Proceedings of the Conference of the American Physical Society Topical Group on Shock Compression of Condensed Matter. AIP Conference Proceedings, Volume 706, pp. 455-460 (2004).

[4] Kirkpatrick, S., Peterson, B., Goetz, R. \& MacNeill, R., IED Fragment/Airblast Threat Module for the Simulation Based Design System, March, 2004.

[5] Butler, L.A., Edwards, E.W., BRL-CAD Tutorial Series: Volume 1 Overview and Installation, ARL-SR-113, U.S. Army Research Laboratory, February, 2002.

[6] Holcomb, J., Young, L.A. \& Needham, C.E., Understanding Injuries Caused by Improvised Explosive Devices in Operation Iraqi Freedom, Draft Report, February 2008.

[7] Yatteau, J.D., Zernow, R.H. \& Recht, R.F., FATEPEN2 Compact Fragment Penetration Model Volume 1 - Model Description, Report No. 5599-2, Applied Research Associates, Inc., January 1991.

[8] Bingham, B.L., Doolittle, C.M., Hacker, W.L., Hikida, S., Plamondon, M.A. \& Whitehouse, S.R., A Code for Blast Effectiveness Against Mobile Systems (BEAMS Version 6.9), Theoretical Manual, prepared by Applied Research Associates, Inc. for U.S. Army Research Laboratory, December 2002.

[9] LS-DYNA Keyword User's Manual, Livermore Software Technology Corporation, Version 970, April 2003.

[10] Young, L.A., Champion H.R. \& Bass C.R., Bioengineering of Blast Injury, prepared for Combating Terrorism Technology Support Office, under Contract F08637-03-C-6006, April 2006.

[11] Bowen I.G., Fletcher E.R. \& Richmond D.R., Estimate of Man's Tolerance to the Direct Effects of Airblast, prepared by Lovelace Foundation for Medical Education and Research for the Defence Atomic Support Agency, DASA-2113, October 1968.

[12] Kokinakis, W. \& Sperrazza, J., Criteria for Incapacitating Soldiers with Fragments and Flechettes, BRL Report No. 1269, Ballistic Research Laboratories, January 1965.

[13] Sturdivan, L.M., Human Vulnerability Modelling for Terrorist Bombing Risk Assessment, Army Research Laboratory Survivability and Lethality Analysis Directorate, December 2002. 